\title{
The Haematopoietic Stem Cell Niche: New Insights into the Mechanisms Regulating Haematopoietic Stem Cell Behaviour
}

\author{
Andrew J. Lilly, ${ }^{1}$ William E. Johnson, ${ }^{2}$ and Christopher M. Bunce ${ }^{1}$ \\ ${ }^{1}$ School of Biosciences, University of Birmingham, Edgbaston, Birmingham, B15 2TT, UK \\ ${ }^{2}$ School of Life and Health Sciences, Aston University, Aston Triangle, Birmingham, B4 7ET, UK \\ Correspondence should be addressed to Christopher M. Bunce, c.m.bunce@bham.ac.uk
}

Received 10 May 2011; Accepted 27 September 2011

Academic Editor: Linheng Li

Copyright ( 2011 Andrew J. Lilly et al. This is an open access article distributed under the Creative Commons Attribution License, which permits unrestricted use, distribution, and reproduction in any medium, provided the original work is properly cited.

\begin{abstract}
The concept of the haematopoietic stem cell (HSC) niche was formulated by Schofield in the 1970s, as a region within the bone marrow containing functional cell types that can maintain HSC potency throughout life. Since then, ongoing research has identified numerous cell types and a plethora of signals that not only maintain HSCs, but also dictate their behaviour with respect to homeostatic requirements and exogenous stresses. It has been proposed that there are endosteal and vascular niches within the bone marrow, which are thought to regulate different HSC populations. However, recent data depicts a more complicated picture, with functional crosstalk between cells in these two regions. In this review, recent research into the endosteal/vascular cell types and signals regulating HSC behaviour are considered, together with the possibility of a single subcompartmentalised niche.
\end{abstract}

\section{Introduction}

Haematopoietic stem cells (HSCs) are a heterogeneous group of multipotent stem cells that have the ability to self-renew and differentiate into all the functional blood cell types of the body. During vertebrate development, the first source of haematopoiesis is extraembryonic haemangioblasts. These cells predominantly generate erythrocytes and endothelial cells to supply the emerging yolk sac vasculature and have different properties to HSCs of the adult which arise later in development [1]. For this review, HSCs will be considered as cells able to confer long-term myeloid and lymphoid multilineage haematopoiesis. Transplantation studies in the mouse have demonstrated that the first HSCs that satisfy this definition emerge during the development of the embryonic blood vessels from a subset of cells called the haemogenic endothelium [2-4]. The HSCs then colonise the fetal liver, where there is HSC expansion and the appearance of characteristic cell surface markers of adult HSCs $[5,6]$. Late in fetal development, HSCs home and engraft in the bone marrow, where they reside throughout adult life [7]. The bone marrow is the main site of adult haematopoiesis, although during times of stress haematopoiesis may also occur in the spleen and liver.
A multitude of studies have identified numerous intrinsic pathways that regulate HSC self-renewal and differentiation programmes [8]. However, in the 1970s, it was noted that whilst HSCs in the bone marrow drive haematopoiesis throughout the life of organisms, when they are removed from the bone marrow, they lose the ability to self renew indicating the equal dependence of HSCs on extrinsic signals [9]. This led Schofield to propose the "niche" hypothesis, which states that HSCs require the support of other cell types in the bone marrow to maintain HSC potency [9]. It is now clear that other than simply maintaining HSCs, the niche plays important roles in regulating the behaviour of HSCs with respect to homeostasis and responses to exogenous stresses. For example, under normal conditions most HSCs in the bone marrow are dormant or slowly cycling, which prevents stem cell exhaustion and maintains haematopoiesis $[10,11]$. However, under periods of haematopoietic stress such as blood loss, HSCs and progenitors are activated to proliferate and differentiate to replace the lost cells [12].

Schofield's recognition of the HSC niche has fostered ongoing research trying to identify and understand the cellular and molecular components that make up the niche. At present, there is broad discussion of the possible presence of two bone marrow niches able to maintain and regulate 
HSCs, which are the endosteal and vascular niches. However, whether these environments truly represent two distinct HSC niches still remains under debate.

\section{The Endosteal Niche}

The endosteum is the interface between bone and bone marrow. This region is lined with a heterogeneous group of osteoblastic cells at various stages of differentiation, only a fraction of which are fully mature osteoblasts able to synthesise bone. Osteoclasts, which are bone-absorbing cells, also line the endosteum and dynamically balance bone formation with the osteoblasts. Several lines of investigation have pointed to the importance of osteoblastic cells in maintaining and supporting HSCs in the niche. The coculture of HSCs and osteoblast cell lines in vitro results in an expansion of HSC numbers indicating enhanced ex vivo self-renewal [13]. Similarly, increasing the number of osteoblastic cells in vivo correlates with an increase in the number of HSCs [14, 15]. In addition, the cotransplantation of osteoblasts with HSCs in mice significantly enhances engraftment [16].

Osteoblastic cells synthesise a number of cytokines that appear to contribute to the maintenance and regulation of HSCs by the endosteal niche. These include thrombopoietin (THPO) and angiopoietin (Ang-1), which bind to cell surface receptors MPL and Tie2, respectively, which are expressed on HSCs. These cytokines are thought to be important as THPO and Ang-1 knockout mice have decreased numbers or defects in bone marrow HSCs [17-19]. In agreement with these data, stimulation of the MPL receptor with THPO enhances the quiescence of long term (LT)-HSCs, whilst inhibition of the receptor decreases their quiescence [20]. The THPO/MPL pathway is thought to promote quiescence by activating cyclin-dependent kinase inhibitors (CDKIs) such as p57 $7^{\text {kip2 }}$ [21]. The interaction of Ang-1 with its receptor Tie2 has also been shown to enhance quiescence and maintain long term repopulating ability of HSCs, whilst protecting against apoptosis by activating the PI3K pathway [22].

Besides secreting important cytokines, osteoblastic cells also express a range of membrane-bound ligands and adhesion receptors which contribute to the maintenance of HSCs within the endosteal niche. Osteoblastic cells express Jagged, a ligand for Notch receptors expressed on HSCs. Activation of Notch receptors on HSCs has been shown to inhibit differentiation and enhance their self renewal capacity in vitro $[23$, 24]. Conversely, in vivo Notch deletion studies failed to show any significant effects of Notch signalling on HSC differentiation and self renewal, making the role of Notch in the niche controversial $[25,26]$. However, it has been recently demonstrated that Notch2 knockout mice have a reduced ability to recover from bone marrow injury induced by 5 fluorouracil (5-FU) treatment as well as a decrease in repopulating HSCs in the bone marrow [27]. This suggests that Notch signalling is important in vivo for controlling HSC self renewal and differentiation during haematopoietic stress conditions and is masked by other factors or is redundant during homeostasis.
$\mathrm{N}$-Cadherin is a calcium-dependent homophilic adhesion molecule [28], which is expressed on both immature and mature osteoblastic cell populations. Like the Jagged/ Notch pathway, the role of N-Cadherin in the endosteal niche remains controversial $[29,30]$. In some mouse studies neither $\mathrm{N}$-Cadherin mRNA nor protein could be detected in HSCs [31], whereas other studies report its expression in both ST-HSCs and LT-HSCs $[32,33]$. The N-Cadherin knock-out mouse has no apparent defects in HSC activity or haematopoiesis, indicating that HSCs do not depend on $\mathrm{N}$-Cadherin for maintenance in vivo [34]. However, HSCs that express a dominant negative mutant $\mathrm{N}$-Cadherin have a reduced ability to anchor to the endosteum and consequently a diminished capacity to repopulate bone marrow following transplantation [32]. Therefore, the role of $\mathrm{N}$-Cadherin in the endosteal niche remains uncertain, but it seems to have a role in enhancing endosteal-HSC interactions following bone marrow transplantation.

Chemokines and their receptors control HSC behaviour by regulating migration, homing, and release of HSCs within the bone marrow. The best-understood chemokine is stromal-derived factor-1 (SDF-1) also called chemokine C$\mathrm{X}-\mathrm{C}$ motif ligand 12 (CXCL12). The SDF-1 receptor is the $\mathrm{C}-\mathrm{X}-\mathrm{C}$ chemokine receptor type 4 (CXCR4) and is expressed on HSCs and progenitors. The importance of SDF-1/CXCR4 signalling has been demonstrated by both $\mathrm{SDF}^{-1^{-/}}$and CXCR4 ${ }^{-/-}$mice, which have severe defects in myelopoiesis, including a decreased number of myeloid progenitors within the bone marrow $[35,36]$. SDF-1 is secreted by a variety of cells within the bone marrow including osteoblasts, endothelial cells, and scattered stromal cells [37], but it has recently been reported that osteoblasts are high secretors of SDF-1 [38]. In agreement with these data the authors found that the HSC-mobilising cytokine G-CSF exerts its long term effects partly by decreasing osteoblast activity resulting in reduced endosteal SDF-1 levels [38].

Although osteoblastic cells are the most studied in terms of HSC maintenance within the endosteal niche, many other types of cells are present. Increasing evidence suggests that bone-degrading osteoclasts play an important role in regulating HSCs within the bone marrow. The degradation of bone by osteoclasts releases factors embedded in the bone matrix. These include transforming growth factor beta 1 (TGF beta 1), bone morphogenic proteins (BMPs), and calcium ions [39-41]. TGF beta 1, BMP-2, and BMP-7 have been demonstrated to enhance the quiescence and maintenance of HSCs in vitro $[39,40]$, whilst evidence suggests that calcium ions enhance the retention of HSCs to the endosteal surface [42]. In support of these data, mice treated with bisphosphonates, which inhibit osteoclast reabsorbing activity, have diminished numbers of LT-HSCs within the bone marrow coupled with reduced HSC quiescence and enhanced differentiation [43].

Accumulating evidence now indicates that tissue-specific macrophages play important roles in supporting the development of a variety of tissues [44]. Macrophages termed osteomacs are found at the endosteum in close proximity to osteoblasts and osteoclasts [45]. Osteomacs regulate osteoblast function and are required for optimal mineralisation 
in vitro and in vivo [45]. Depletion of osteomacs in vivo also results in decreased numbers of osteoblasts and a reduction in osteoblast-secreted cytokines such as Ang-1, KIT ligand, and CXCL12 [46]. These changes are accompanied by the mobilisation of HSCs from the bone marrow, indicating a central role for osteomacs in maintaining the structure and function of the endosteal niche [46]. An important unanswered question is whether osteomacs interact directly with HSCs within the endosteal niche.

\section{Sympathetic Innervation of the HSC Niche}

A complex organisation of neuronal fibres are found within the bone marrow, and sympathetic nervous system (SNS) activity has been reported to control bone formation [47, 48]. However, more recently, a potential role of SNS activity in the endosteal niche has been identified. Frenette and colleagues demonstrated that sulphogalactoceramide (sulfatide), a sulpholipid synthesised by ceramide galactosytransferase (CGT) in neuronal Schwann cells, could mobilise HSCs from the bone marrow [49]. To investigate the effects of sulfatide depletion on the endosteal niche and HSC mobilisation, a CGT knockout mouse was created. As expected, $\mathrm{CGT}^{-/-}$mice showed hematopoietic defects and decreased HSC mobilisation in response to G-CSF administration, but unexpectedly this was not directly due to impaired sulfatide release $[50,51]$. Rather, the decreased ability of the $\mathrm{CGT}^{-/-}$ mice to mobilise HSCs in response to G-CSF was found to be due to impairment of the neuronal signals that regulate osteoblast function [51]. The authors went on to demonstrate that sympathetic nervous system activity suppresses osteoblast function resulting in enhanced HSC mobilisation from the bone marrow [51].

\section{The Vascular Niche}

Several lines of evidence suggest that vascular environments are involved in the maintenance of HSCs as well as endosteal environments. During fetal development, fetal functioning HSCs first arise from the haemogenic endothelium of the vasculature, indicating that the two tissues are developmentally closely related [2-4]. Fetal HSCs then reside in the liver and the spleen, where early haematopoiesis takes place in the absence of osteoblasts or the endosteal niche [5, 6]. Even in adults, HSCs are present in the liver and the spleen throughout life and are capable of extramedullary haematopoiesis $[52,53]$. These observations indicate that cells of vascular environments can both support HSCs and regulate their self renewal and differentiation.

The bone marrow is heavily vascularised; the medullary artery feeds in to arterioles, capillaries, and then the sinusoids. Sinusoids are specialised blood vessels, which have thin walls with a fenestrated arrangement of endothelial cells to allow the passage of haematopoietic cells [54]. The sinusoids form an extensive network throughout the bone marrow, and therefore the endothelial cells which make up the sinusoids are important when considering HSC bone marrow niche. This idea has been supported by mouse endothelial cell and
HSC co-culture experiments, which demonstrate that some endothelial cell populations can support the expansion of HCSs in vitro $[55,56]$. As well as this, some endothelial cell populations can maintain severe combined immunedeficient (SCID) mouse repopulating capacity of HSCs during co-culture, $[57,58]$.

Important recent work indicates that endothelial cells are important for haematopoiesis in vivo. Yao and colleagues sought to investigate whether the reduced numbers of HSCs and defects in haematopoiesis of gp130 knockout mice [59] were directly due to effects on HSCs or due to effects on niche endothelial cells [60]. Mice expressing Cre recombinase under control of the Tie2 promoter, which is active in HSCs and endothelial cells, were crossed with mice containing a gp130 gene with loxP sites. The resulting mice had a conditional deletion of $g p 130$ in both HSCs and endothelial cells. These mice appeared normal at birth but had hypocellular bone marrow, developed expanded sinusoidal spaces, and died prematurely at around 1 year. Transplantation of bone marrow from gp130 deficient mice into normal irradiated mice restored normal haematopoiesis. However, transplantation of bone marrow from normal mice into gp130-deficient irradiated mice did not restore normal haematopoiesis. These complimentary transplantation experiments indicate that endothelial cells are essential components of the HSC niche and that endothelial gp130 signalling is crucial for maintaining haematopoiesis [60].

Other evidence indicates that many HSCs are located near the sinusoids in the bone marrow. It has been suggested that the rapid mobilisation of HSCs within minutes of administering G-CSF argues that a large proportion of HSCs must be very close to blood vessels $[61,62]$. More recently, Kiel and colleagues identified a group of markers called signalling lymphocyte activation molecule (SLAM) family markers which include CD150, CD244, and CD48, which are differentially expressed between HSC and progenitor subpopulations in mice [63]. The most immature HSCs could be precisely identified as CD150 ${ }^{+v e}, \mathrm{CD} 244^{-\mathrm{ve}}$, and $\mathrm{CD} 48^{-\mathrm{ve}}$, allowing reliable imaging of HSCs within the bone marrow for the first time $[31,63]$. Strikingly, they found that around $60 \%$ of HSCs defined in this way were in contact with the endothelium of sinusoids, whereas, only around $20 \%$ were found at the endosteal surface. Overall only $10 \%$ of the total bone marrow nucleated cells were found at the sinusoid endothelium, indicating a 6-fold selective enrichment of HSCs in this area. Whilst these results point to a distinct vascular niche for HSCs within the bone marrow, virtually all HSCs (92-95\%) were also found within 5 cell diameters from the sinusoid endothelium. Thus, it remains possible that HSCs at the endosteum are also effected by vascular cells [31].

Reticular cells are a group of cells that are located around the sinusoid endothelium and are important in the homing and localisation of HSCs within the bone marrow. These cells have recently been shown to be high secretors of SDF-1 (CXCL12), and as a result have been named CXCL12 abundant reticular (CAR) cells [64]. By using immunohistochemical analysis of the bone marrow of CXCL12-GFP knock in mice, Sugiyama and colleagues have shown that nearly all 
(97\%) of HSCs within the bone marrow were localized to CAR cells. Interestingly, $100 \%$ of the HSCs located at the endosteum were also located to CAR cells. These data suggest that CAR cells are crucial for the homing of HSCs in both the vascular and endosteal niches and together with the data of Kiel and colleagues, indicate that functionally there may be just one class of niche in the bone marrow.

Further data from Omatsu et al. (2010) suggests that CAR cells are important in supporting the proliferation of HSCs/progenitors as selective ablation of CAR cells resulted in a reduction in the number of cycling progenitors, and enhanced the quiescence of HSCs in the bone marrow [65]. As well as this, CAR cells were found to be high secretors of the proproliferative cytokine stem cell factor (SCF), and that CAR cell ablation resulted in a decreased in SCF levels [65]. The authors went on to show that CAR cells express adipogenic and osteogenic genes and can differentiate into adipocytes and osteoblasts in vitro. These data suggest that CAR cells are a form of adipo-osteogenic progenitor derived from mesenchymal stem cells [65]. Mesenchymal stem cell (MSC) was a term proposed by Caplan in 1991 [66] to describe the multipotent bone marrow stromal cell populations, which Owen and Friedenstein demonstrated in the 1960s and 1970s to be capable of undergoing osteoblastic differentiation to form bone [67]. Whether MSCs truly represent a stem cell capable of self renewal and multipotency on a single cell basis is not proven in vivo [68]. However, their participation in supporting the vascular HSC niche is better established, specifically CD $146^{\text {+ve }}$ cells that are found in the subendothelial layers of sinusoids [69]. Bone marrow-derived CD146 ${ }^{\text {tve }}$ cells are capable of differentiating in vitro along multiple lineages to form osteoblasts, chondrocytes, and adipocytes, which is considered characteristic of MSCs [70], but only CD $146^{\text {tve }}$ cells (and not other MSC-like populations) have been demonstrated conclusively and clonally to recapitulate the haematopoietic microenvironment and synthesise bone when transplanted heterotopically [69]. HSCs are also better supported in vitro by MSCs derived from bone marrow than MSCs derived from other tissues, for example from adipose tissue [71]. CD146 ${ }^{\text {tve }}$ bone marrow cells express a variety of factors that support HSCs, including Jagged-1, N cadherin, stem cell factor (SCF), and SDF-1 [69].

Megakaryocytes have been implicated to be involved in regulating haematopoiesis, since mice with decreased mature megakaryocytes and platelets have other haematopoietic defects such as anaemia and myelofibrosis [72, 73]. However, the contribution of megakaryocytes in regulating HSCs by direct or indirect mechanisms is poorly understood. Megakaryocytes are located close to sinusoidal endothelial cells in the perivascular environment $[74,75]$, and data also suggests that they communicate with the endosteal niche. Co-culture of megakaryocytes and osteoblasts in vitro enhances osteoblast proliferation up to 6 -fold, a mechanism requiring direct cell contact [76-78]. These data are supported by in vivo studies, using NF-E2 knockout mice, which have an accumulation of megakaryocytes and an increase in bone mass and osteoblasts [76, 79]. Kacena and colleagues [80] transplanted spleen cells from NF-E2 ${ }^{-/-}$mice into irradiated mice and after 4 weeks found the same megakaryocytic and osteoblastic phenotype as the donor NF-E2 ${ }^{-/-}$mice. These data suggest that the increase in bone mass and osteoblasts seen in the NF-E2 $2^{-/}$mice is an indirect feature, caused by an increase in megakaryocytes [80].

Recent data suggests that megakaryocytes and platelets directly regulate the behaviour of HSCs. Like HSCs, megakaryocytes and their precursors express $\mathrm{Mpl}$, the receptor for TPO, and as the name thrombopoietin suggests, signalling via this pathway is necessary for megakaryocytic differentiation and platelet formation $[81,82]$. Serum TPO levels inversely correlate with megakaryocyte mass suggesting that TPO levels are regulated by the amount of cells which can take up and remove TPO from the circulation via $\mathrm{Mpl}[83,84]$. This provokes an interesting question whether megakaryocytes regulate the levels of available TPO and consequently regulate HSC quiescence. De Graaf and colleagues [85] investigated this possibility by creating $m y b$ and $p 300$ mutations in mice that lead to an increase in megakaryocytes and platelets. As expected, these mutants had decreased serum TPO levels, and a striking change in TPO responsive genes in bone marrow HSCs. Furthermore, the authors found that this resulted in increased HSC cycling consistent with the decreased TPO levels. The authors conclude that the TPO regulates megakaryocytic differentiation, which in turn regulates circulating TPO levels and quiescence of HSCs [85]. This feedback mechanism could be important to maintain homeostasis during injury. For example, increased bleeding would cause a decrease in platelets resulting in an increase in circulating TPO. The increased TPO could then not only drive differentiation of megakaryocytic precursors to replace the lost platelets, but also enhance the quiescence of LT-HSCs to prevent stem cell exhaustion and protect future haematopoiesis. These data provide exciting new evidence that HSCs are regulated by their mature progeny, a process which has been poorly studied but has to be important to guide haematopoiesis with respect to the levels of mature cell populations.

\section{Evidence for an Adult Haemangioblast}

The relationship and proximity of HSCs to sinusoidal endothelial cells provoke the question whether adults have haemangioblasts and haemogenic endothelial cells which can renew HSC within adult bone marrow. In humans, CD34 is a marker expressed on both HSCs and endothelial progenitor cell populations. HSCs and endothelial progenitors are further enriched within the CD34 ${ }^{\text {tve }}$ and vascular endothelial growth factor receptor $2\left(\mathrm{KDR}^{+\mathrm{ve}}\right)$ population, whilst HSCs only are found within the $\mathrm{CD} 34^{+\mathrm{ve}} / \mathrm{KDR}^{-\mathrm{ve}}$ fraction [86]. Pelosi and colleagues used limiting dilution assays to show that $5 \%$ of cells within the $\mathrm{CD} 34^{+\mathrm{ve}} / \mathrm{KDR}^{+\mathrm{ve}}$ fraction could give rise to both haematopoietic and endothelial lineages, indicative of haemangioblast activity [86]. Coupled with this, it has been suggested that some HSCs expressing CD34 and leukocyte marker CD45 have haemangioblast activity in vivo, as transplantation of these cells into mice results in donorderived vascularisation [87]. However, the transplantation of a small contaminating population of endothelial progenitors 
cannot be fully ruled out in this study. These studies are supported by investigations in chronic myeloid leukaemia (CML), a clonal malignancy arising in the HSC compartment, characterised by expression of the BCR:ABL fusion gene. Endothelial cells from CML patients have been found that express the BCR:ABL fusion gene, suggesting that endothelial cells and HSCs have a common ancestor [88]. However, the location of the adult haemangioblasts within the bone marrow niche is unknown, as well as its contribution to renewing the HSC pool. Also, unlike in the embryo, an adult haemogenic endothelium with the potential to give rise to HSCs has not yet been discovered.

\section{The Role of Small Bioactive Signalling Molecules in the HSC Niche}

The majority of research into signalling mechanisms within the bone marrow microenvironment has focussed on protein molecules such as cytokines, chemokines, adhesion molecules, and their prospective receptors. However, small nonprotein bioactive molecules such as eicosanoids are emerging as essential signalling mediators regulating HSC behaviour within the niche. Eicosanoids are a group of 20 carbon fatty acids that are derived from arachidonc acid and include; prostacyclins, prostaglandins, thromboxanes, endocannabinoids, and leukotrienes. Eicosanoids are synthesised by a wide range of cells and have diverse effects [89].

Prostaglandins are the best understood eicosanoids, and of which prostaglandin E2 (PGE2) is the most studied in terms of modulating HSC behaviour. PGE2 synthesis is a two-step process; COX-1 and COX-2 enzymes convert arachidonic acid into prostaglandin $\mathrm{H} 2$ (PGH2), which is further converted to PGE2 by prostaglandin synthases. PGE2 is secreted by efflux transporters called multi-drug resistance proteins (MDRP), where it can act as an autocrine or paracrine signal by binding to G-protein-coupled E prostanoid (EP) receptors. Four different EP receptors for PGE2 have been identified (EP1 to EP4) [90], and all four of these have been shown to be expressed on both murine and human HSCs [91]. Evidence suggests that PGE2 is secreted by a variety of cell types which are found in the bone marrow including; osteoblasts [92-94], monocytes/macrophages [95, 96], and sinusoidal endothelial cells $[97,98]$. Therefore, PGE2 is likely to be found in both endosteal and vascular niches.

In seminal studies from North et al. [99], a chemical genetic screen was used to identify important factors regulating HSC formation during zebrafish embryogenesis. It was found that chemicals that increased PGE2 synthesis, such as linoleic acid, also increased HSC numbers, whilst COX inhibitors, such as celecoxib, decreased HSC numbers. In agreement with the screen, exogenous use of a stable derivative of PGE2, 16,16-dimethyl-PGE2 (dmPGE2), increased HSC formation during embryogenesis and enhanced the haematopoietic recovery of irradiated adult zebrafish. In murine models, treatment of HSCs with dmPGE2 in vitro also enhanced HSC repopulating capacity following transplantation into irradiated mice [99]. Follow-up experiments demonstrated that PGE2 signalling enhanced HSC wnt signalling associated with increased HSC proliferation [100]. Others found that PGE2 increased the expression of survivin in HSCs, an antiapoptotic protein which enhances HSC survival as well as progression through cell cycle [91]. In addition to this, PGE2 has been found to increase CXCR4 expression on HSCs, thereby enhancing migration to SDF-1 and homing to the bone marrow $[91,101]$.

Although these studies provide some of the first enticing evidence of the role of PGE2 in the HSC niche, surprisingly little is known about the involvement of other eicosanoids. Data suggests that prostaglandin D2 (PGD2), another derivative of $\mathrm{PGH} 2$, is the major prostaglandin in the bone marrow [102], but its effects on HSCs remain largely uninvestigated. Leukotriene synthesis has also been shown in the bone marrow [103], and there is evidence that leukotrienes can stimulate myeloid progenitor proliferation in vitro [104]. Therefore, further research is needed to shed light on the role of the array of small bioactive signalling molecules within the HSC niche.

\section{The Role of Hypoxia in the HSC Niche}

As well as the cells and other factors that make up bone marrow microenvironments, other physiological characteristics of the niche need to be considered, of which hypoxia is emerging as vital in regulating HSC behaviour. The oxygen levels of the bone marrow vary considerably, as areas close to sinusoids are highly perfused, whereas areas further away around the endosteum are poorly perfused and hypoxic. In vitro studies demonstrated that haematopoiesis is enhanced by low oxygen levels of around 1\%, giving suggestive evidence that this may be the case in vivo $[105,106]$. In agreement with these data, mathematical modelling approaches pointed to hypoxic environments for the location of HSCs in the bone marrow [107].

Until recently, the study of hypoxia in the bone marrow was hampered by its complex organisation and inaccessibility. Parmar and colleagues 2007 [108], used Hoechst dye (a DNA-intercalating dye that is readily taken up by live cells) to measure the perfusion of cell populations within the bone marrow. Mice were intravenously infused with a pulse of Hoechst, and then uptake into cells in the bone marrow was measured using flow cytometry. They found a striking broad continuum in the amount of Hoechst taken up with variability in the range of 1000-fold. Furthermore, cells that had taken up the least amount of Hoechst exhibited HSC-like properties in long-term culture initiating assays and highest engraftment frequency in irradiated mice [108].

Winkler et al. [109] built on the above results by analysing not only Hoechst perfusion but also the cell surface markers expressed on HSCs and on more mature progenitors. As expected, they found that the most immature HSCs were Hoechst negative and only these cells could reconstitute haematopoietic system in serial transfer experiments [109]. As only dormant HSCs are able to serially engraft [110], it suggests that HSCs away from sinusoid perfusion contain the most potent and dormant HSCs. The authors also found a phenotypically identical (by surface marker expression) 


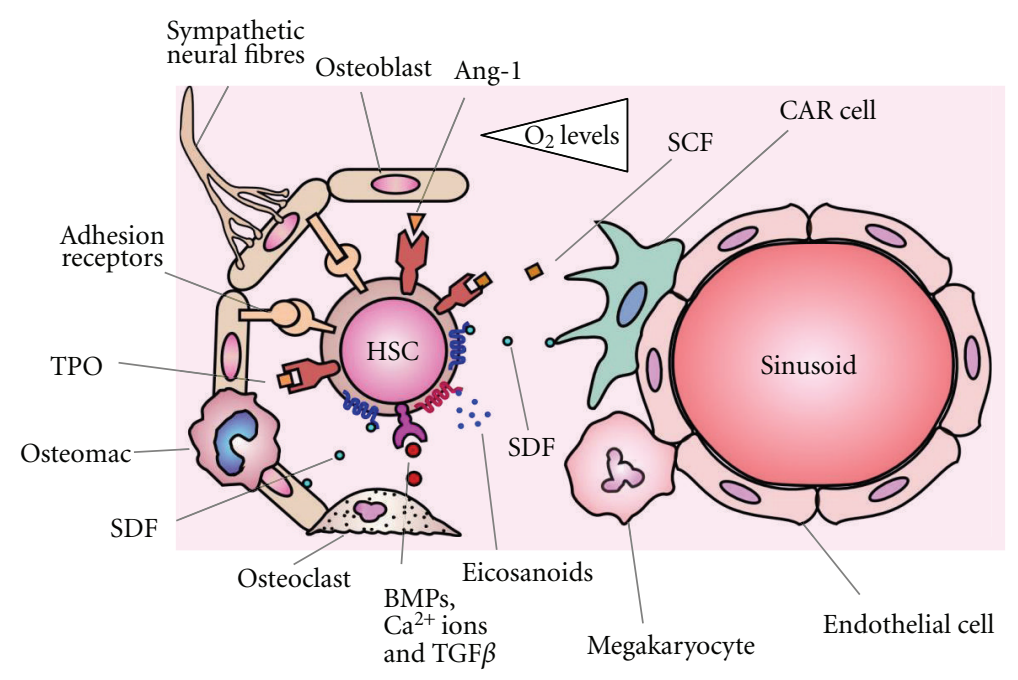

FIGURE 1: Endosteal and vascular niches are subcompartments of a single niche. HSCs located at the endosteum are more quiescent and have a greater self-renewal capacity due to a variety of cytokines, adhesion molecules, and hypoxia. HSCs located close to the sinusoid endothelium have reduced self-renewal capacity and are cycling more rapidly, due to higher oxygen levels and SCF. However, nearly all HSCs reside adjacent to CAR cells and in close proximity to the sinusoid endothelium. The cells of the vascular niche communicate with cells of the endosteal niche, and the subtle balance of factors from these subcompartments governs the behaviour of the HSCs.

population of Hoechst medium cells, which had ability to reconstitute haematopoiesis only in primary recipients [109].

The association of the more potent HSCs with a hypoxic environment may be linked to protection from reactive oxygen species (ROS). When oxygen is metabolised, ROS are released as a byproduct, causing DNA damage and protein miss folding, which have been associated with ageing [111]. Elevating ROS in the haematopoietic system, by creation of ataxia-telangiectasia-mutated-(ATM-) knockout mice, results in progressive bone marrow failure [112]. In agreement with these data, HSCs with low ROS levels have been shown to maintain potency throughout serial transplantation experiments, when compared to HSCs with high ROS levels [113]. Other than causing cellular damage, data suggests that ROS activates the P38 MAPK pathway, which decreases adhesion molecule expression and enhances cell cycle progression $[112,114,115]$. Collectively, these data indicate that hypoxia in the endosteal niche protects HSCs against ROS-induced damage, whilst maintaining nicheHSC interactions and quiescence. This is necessary to maintain HSC self renewal capacity and protect against stem cell exhaustion, whilst the higher oxygen levels in the vascular niche may facilitate progenitor proliferation.

\section{Concluding Remarks}

More than 30 years of research has uncovered an astonishingly complex array of signals within the bone marrow niche, the subtle balance of which dictates the behaviour of HSCs. Distinct endosteal and vascular environments have been identified, where generally speaking the endosteal niche supports quiescent HSCs, whereas the vascular niche facilitates the proliferation of more mature progenitors. However, even HSCs at the endosteum reside within 5 cell diameters of sinusoids [31], and virtually all endosteal HSCs are adjacent to CAR cells associated with the sinusoid endothelium [64]. Coupled with this, the endosteal niche cannot maintain adequate haematopoiesis in mice where the vascular niche has been compromised [60], supporting the accumulating data describing crosstalk and overlap between cells in these two compartments. Consequently, these data reveal that the endosteal/vascular two niches hypothesis is far too basic and possibly should be thought of as subcompartments of a single niche (Figure 1). The advent of novel technologies that now permit the study of the niche in three dimensions and in real time will no doubt accelerate the solution of this problem $[116,117]$. Even so, there is still much to learn about the HSC niche with new avenues including, the role of maturing cells in guiding haematopoiesis with respect to biological need, the effect of small bioactive molecules on HSCs, and functions of the adult haemangioblasts in replenishing the HSC pool.

The knowledge gained from studying the HSC niche has important implications for health and disease. The use of cord blood HSCs to treat disorders in adults has been hampered by their relatively small numbers and shortcomings in expansion techniques of current ex vivo culture systems. Further understanding of the factors involved in regulating self renewal and proliferation of HSCs in the niche, would allow these to be mimicked in vitro, thereby enhancing expansion of HCS prior to transplantation. On the other side of the coin, the quiescence of leukaemic stem cells (LSCs) renders them largely unresponsive to current chemotherapeutic agents, leading to disease relapse. Understanding the myriad factors regulating HSC quiescence and how these signals are perturbed in leukaemia are therefore important to enable the future development of therapies to eradicate LSCs. 


\section{References}

[1] C. Lancrin, P. Sroczynska, A. G. Serrano et al., "Blood cell generation from the hemangioblast," Journal of Molecular Medicine, vol. 88, no. 2, pp. 167-172, 2009.

[2] C. Lancrin, P. Sroczynska, C. Stephenson, T. Allen, V. Kouskoff, and G. Lacaud, "The haemangioblast generates haematopoietic cells through a haemogenic endothelium stage," Nature, vol. 457, no. 7231, pp. 892-895, 2009.

[3] M. J. Chen, T. Yokomizo, B. M. Zeigler, E. Dzierzak, and N. A. Speck, "Runxl is required for the endothelial to haematopoietic cell transition but not thereafter," Nature, vol. 457, no. 7231, pp. 887-891, 2009.

[4] M. Iacovino, D. Chong, I. Szatmari et al., "HoxA3 is an apical regulator of haemogenic endothelium," Nature Cell Biology, vol. 13, no. 1, pp. 72-78, 2011.

[5] S. J. Morrison, H. D. Hemmati, A. M. Wandycz, and I. L. Weissman, "The purification and characterization of fetal liver hematopoietic stem cells," Proceedings of the National Academy of Sciences of the United States of America, vol. 92, no. 22, pp. 10302-10306, 1995.

[6] A. Cumano and I. Godin, "Ontogeny of the hematopoietic system," Annual Review of Immunology, vol. 25, pp. 745-785, 2007.

[7] J. L. Christensen, D. E. Wright, A. J. Wagers, and I. L. Weissman, "Circulation and chemotaxis of fetal hematopoietic stem cells," PLoS Biology, vol. 2, no. 3, article E75, 2004.

[8] M. R. Warr, E. M. Pietras, and E. Passegue, "Mechanisms controlling hematopoietic stem cell functions during normal hematopoiesis and hematological malignancies," Wiley Interdisciplinary Reviews, vol. 3, no. 6, pp. 681-701, 2011.

[9] R. Schofield, "The relationship between the spleen colonyforming cell and the haemopoietic stem cell. A hypothesis," Blood Cells, vol. 4, no. 1-2, pp. 7-25, 1978.

[10] G. B. Bradford, B. Williams, R. Rossi, and I. Bertoncello, "Quiescence, cycling, and turnover in the primitive hematopoietic stem cell compartment," Experimental Hematology, vol. 25, no. 5, pp. 445-453, 1997.

[11] S. H. Cheshier, S. J. Morrison, X. Liao, and I. L. Weissman, "In vivo proliferation and cell cycle kinetics of long-term self-renewing hematopoietic stem cells," Proceedings of the National Academy of Sciences of the United States of America, vol. 96, no. 6, pp. 3120-3125, 1999.

[12] S. H. Cheshier, S. S. Prohaska, and I. L. Weissman, "The effect of bleeding on hematopoietic stem cell cycling and selfrenewal," Stem Cells and Development, vol. 16, no. 5, pp. 707717, 2007.

[13] R. S. Taichmana, M. J. Reilly, and S. G. Emerson, "Osteoblasts and the hematopoietic microenvironment," Hematology, vol. 4, no. 5, pp. 421-426, 2000.

[14] L. M. Calvi, G. B. Adams, K. W. Weibrecht et al., "Osteoblastic cells regulate the haematopoietic stem cell niche," Nature, vol. 425, no. 6960, pp. 841-846, 2003.

[15] J. Zhang, C. Niu, L. Ye et al., "Identification of the haematopoietic stem cell niche and control of the niche size," Nature, vol. 425, no. 6960, pp. 836-841, 2003.

[16] N. S. El-Badri, B. Y. Wang, Cherry, and R. A. Good, "Osteoblasts promote engraftment of allogeneic hematopoietic stem cells," Experimental Hematology, vol. 26, no. 2, pp. 110-116, 1998.

[17] H. Qian, N. Buza-Vidas, C. D. Hyland et al., "Critical role of thrombopoietin in maintaining adult quiescent qematopoietic stem cells," Cell Stem Cell, vol. 1, no. 6, pp. 671-684, 2007.
[18] M. C. Puri and A. Bernstein, "Requirement for the TIE family of receptor tyrosine kinases in adult but not fetal hematopoiesis," Proceedings of the National Academy of Sciences of the United States of America, vol. 100, no. 22, pp. 12753-12758, 2003.

[19] S. Kimura, A. W. Roberts, D. Metcalf, and W. S. Alexander, "Hematopoietic stem cell deficiencies in mice lacking c-Mpl, the receptor for thrombopoietin," Proceedings of the National Academy of Sciences of the United States of America, vol. 95, no. 3, pp. 1195-1200, 1998.

[20] H. Yoshihara, F. Arai, K. Hosokawa et al., “Thrombopoietin/ MPL signaling regulates hematopoietic stem cell quiescence and interaction with the osteoblastic niche," Cell Stem Cell, vol. 1, no. 6, pp. 685-697, 2007.

[21] F. Arai, H. Yoshihara, K. Hosokawa et al., "Niche regulation of hematopoietic stem cells in the endosteum: the role of thrombopoietinmpl signaling in the maintenance of quiescent hematopoietic stem cells," Annals of the New York Academy of Sciences, vol. 1176, pp. 36-46, 2009.

[22] F. Arai, A. Hirao, M. Ohmura et al., "Tie2/angiopoietin-1 signaling regulates hematopoietic stem cell quiescence in the bone marrow niche," Cell, vol. 118, no. 2, pp. 149-161, 2004.

[23] B. Varnum-Finney, L. Xu, C. Brashem-Stein et al., "Pluripotent, cytokine-dependent, hematopoietic stem cells are immortalized by constitutive Notch1 signaling," Nature Medicine, vol. 6, no. 11, pp. 1278-1281, 2000.

[24] J. M. Butler, D. J. Nolan, E. L. Vertes et al., "Endothelial cells are essential for the self-renewal and repopulation of notchdependent hematopoietic stem cells," Cell Stem Cell, vol. 6, no. 3, pp. 251-264, 2010.

[25] I. Maillard, U. Koch, A. Dumortier et al., "Canonical notch signaling is dispensable for the maintenance of adult hematopoietic stem cells," Cell Stem Cell, vol. 2, no. 4, pp. 356-366, 2008.

[26] S. J. C. Mancini, N. Mantei, A. Dumortier, U. Suter, H. R. MacDonald, and F. Radtke, "Jagged1-dependent Notch signaling is dispensable for hematopoietic stem cell self-renewal and differentiation," Blood, vol. 105, no. 6, pp. 2340-2342, 2005.

[27] B. Varnum-Finney, L. M. Halasz, M. Sun, T. Gridley, F. Radtke, and I. D. Bernstein, "Notch2 governs the rate of generation of mouse long- and short-term repopulating stem cells," Journal of Clinical Investigation, vol. 121, no. 3, pp. 1207-1216, 2011.

[28] M. Takeichi, K. Hatta, A. Nose, and A. Nagafuchi, "Identification of a gene family of cadherin cell adhesion molecules," Cell Differentiation and Development, vol. 25, supplement, pp. 91-94, 1988.

[29] P. Li and L. I. Zon, "Resolving the controversy about N-cadherin and hematopoietic stem cells," Cell Stem Cell, vol. 6, no. 3, pp. 199-202, 2010.

[30] K. Hosokawa, F. Arai, H. Yoshihara et al., "Knockdown of $\mathrm{N}$-cadherin suppresses the long-term engraftment of hematopoietic stem cells," Blood, vol. 116, no. 4, pp. 554-563, 2010.

[31] M. J. Kiel, G. L. Radice, and S. J. Morrison, "Lack of evidence that hematopoietic stem cells depend on N-cadherinmediated adhesion to osteoblasts for their maintenance," Cell Stem Cell, vol. 1, no. 2, pp. 204-217, 2007.

[32] K. Hosokawa, F. Arai, H. Yoshihara et al., "Cadherin-based adhesion is a potential target for niche manipulation to protect hematopoietic stem cells in adult bone marrow," Cell Stem Cell, vol. 6, no. 3, pp. 194-198, 2010. 
[33] J. S. Haug, X. C. He, J. C. Grindley et al., "N-Cadherin expression level distinguishes reserved versus primed states of hematopoietic stem cells," Cell Stem Cell, vol. 2, no. 4, pp. 367-379, 2008.

[34] M. J. Kiel, M. Acar, G. L. Radice, and S. J. Morrison, "Hematopoietic stem cells do not depend on N-cadherin to regulate their maintenance," Cell Stem Cell, vol. 4, no. 2, pp. 170-179, 2009.

[35] T. Nagasawa, S. Hirota, K. Tachibana et al., "Defects of B-cell lymphopoiesis and bone-marrow myelopoiesis in mice lacking the CXC chemokine PBSF/SDF-1," Nature, vol. 382, no. 6592, pp. 635-638, 1996.

[36] Y. R. Zou, A. H. Kottman, M. Kuroda, I. Taniuchi, and D. R. Littman, "Function of the chemokine receptor CXCR4 in heaematopolesis and in cerebellar development," Nature, vol. 393, no. 6685, pp. 595-599, 1998.

[37] I. Petit, M. Szyper-Kravitz, A. Nagler et al., "G-CSF induces stem cell mobilization by decreasing bone marrow SDF- 1 and up-regulating CXCR4," Nature Immunology, vol. 3, no. 7, pp. 687-694, 2002.

[38] C. L. Semerad, M. J. Christopher, F. Liu et al., "G-CSF potently inhibits osteoblast activity and CXCL12 mRNA expression in the bone marrow," Blood, vol. 106, no. 9, pp. 3020-3027, 2005.

[39] P. Batard, M. N. Monier, N. Fortunel et al., "TGF- $\beta 1$ maintains hematopoietic immaturity by a reversible negative control of cell cycle and induces CD34 antigen up-modulation," Journal of Cell Science, vol. 113, no. 3, pp. 383-390, 2000.

[40] M. Bhatia, D. Bonnet, D. Wu et al., "Bone morphogenetic proteins regulate the developmental program of human hematopoietic stem cells," Journal of Experimental Medicine, vol. 189, no. 7, pp. 1139-1147, 1999.

[41] I. A. Silver, R. J. Murrills, and D. J. Etherington, "Microelectrode studies on the acid microenvironment beneath adherent macrophages and osteoclasts," Experimental Cell Research, vol. 175, no. 2, pp. 266-276, 1988.

[42] G. B. Adams, K. T. Chabner, I. R. Alley et al., "Stem cell engraftment at the endosteal niche is specified by the calciumsensing receptor," Nature, vol. 439, no. 7076, pp. 599-603, 2006.

[43] S. Lymperi, A. Ersek, F. Ferraro, F. Dazzi, and N. J. Horwood, "Inhibition of osteoclast function reduces hematopoietic stem cell numbers in vivo," Blood, vol. 117, no. 5, pp. 15401549, 2011.

[44] J. W. Pollard, "Trophic macrophages in development and disease," Nature Reviews Immunology, vol. 9, no. 4, pp. 259270, 2009.

[45] M. K. Chang, L. J. Raggatt, K. A. Alexander et al., "Osteal tissue macrophages are intercalated throughout human and mouse bone lining tissues and regulate osteoblast function in vitro and in vivo," Journal of Immunology, vol. 181, no. 2, pp. 1232-1244, 2008.

[46] I. G. Winkler, N. A. Sims, A. R. Pettit et al., "Bone marrow macrophages maintain hematopoietic stem cell (HSC) niches and their depletion mobilizes HSCs," Blood, vol. 116, no. 23, pp. 4815-4828, 2010.

[47] S. Takeda, F. Elefteriou, R. Levasseur et al., "Leptin regulates bone formation via the sympathetic nervous system," Cell, vol. 111, no. 3, pp. 305-317, 2002.

[48] M. Bliziotes, S. McLoughlin, M. Gunness, F. Fumagalli, S. R. Jones, and M. G. Caron, "Bone histomorphometric and biomechanical abnormalities in mice homozygous for deletion of the dopamine transporter gene," Bone, vol. 26, no. 1, pp. 15-19, 2000.

[49] P. S. Frenette and L. Weiss, "Sulfated glycans induce rapid hematopoietic progenitor cell mobilization: evidence for selectin-dependent and independent mechanisms," Blood, vol. 96, no. 7, pp. 2460-2468, 2000.

[50] Y. Katayama and P. S. Frenette, "Galactocerebrosides are required postnatally for stromal-dependent bone marrow lymphopoiesis," Immunity, vol. 18, no. 6, pp. 789-800, 2003.

[51] Y. Katayama, M. Battista, W. M. Kao et al., "Signals from the sympathetic nervous system regulate hematopoietic stem cell egress from bone marrow," Cell, vol. 124, no. 2, pp. 407-421, 2006.

[52] H. Taniguchi, T. Toyoshima, K. Fukao, and H. Nakauchi, "Presence of hematopoietic stem cells in the adult liver," Nature Medicine, vol. 2, no. 2, pp. 198-203, 1996.

[53] Y. Morita, A. Iseki, S. Okamura, S. Suzuki, H. Nakauchi, and H. Ema, "Functional characterization of hematopoietic stem cells in the spleen," Experimental Hematology, vol. 39, no. 3, pp. 351.e3-359.e3, 2011.

[54] M. J. Kiel and S. J. Morrison, "Uncertainty in the niches that maintain haematopoietic stem cells," Nature Reviews Immunology, vol. 8, no. 4, pp. 290-301, 2008.

[55] J. E. Cardier and E. Barberá-Guillem, "Extramedullary hematopoiesis in the adult mouse liver is associated with specific hepatic sinusoidal endothelial cells," Hepatology, vol. 26, no. 1, pp. 165-175, 1997.

[56] W. Li, S. A. Johnson, W. C. Shelley, and M. C. Yoder, "Hematopoietic stem cell repopulating ability can be maintained in vitro by some primary endothelial cells," Experimental Hematology, vol. 32, no. 12, pp. 1226-1237, 2004.

[57] J. P. Chute, G. Muramoto, J. Fung, and C. Oxford, "Quantitative analysis demonstrates expansion of SCID-repopulating cells and increased engraftment capacity in human cord blood following ex vivo culture with human brain endothelial cells," Stem Cells, vol. 22, no. 2, pp. 202-215, 2004.

[58] X. Cheng, T. Macvittie, B. Meisenberg et al., "Human brain endothelial cells (HUBEC) promote SCID repopulating cell expansion through direct contact," Growth Factors, vol. 25, no. 3, pp. 141-150, 2007.

[59] K. Yoshida, T. Taga, M. Saito et al., "Targeted disruption of gp130, a common signal transducer for the interleukin 6 family of cytokines, leads to myocardial and hematological disorders," Proceedings of the National Academy of Sciences of the United States of America, vol. 93, no. 1, pp. 407-411, 1996.

[60] L. Yao, T. Yokota, L. Xia, P. W. Kincade, and R. P. McEver, "Bone marrow dysfunction in mice lacking the cytokine receptor gp130 in endothelial cells," Blood, vol. 106, no. 13, pp. 4093-4101, 2005.

[61] L. Laterveer, I. J. D. Lindley, M. S. Hamilton, R. Willemze, and W. E. Fibbe, "Interleukin-8 induces rapid mobilization of hematopoietic stem cells with radioprotective capacity and long-term myelolymphoid repopulating ability," Blood, vol. 85, no. 8, pp. 2269-2275, 1995.

[62] B. Heissig, K. Hattori, S. Dias et al., "Recruitment of stem and progenitor cells from the bone marrow niche requires MMP9 mediated release of Kit-ligand," Cell, vol. 109, no. 5, pp. 625-637, 2002.

[63] M. J. Kiel, Ö. H. Yilmaz, T. Iwashita, C. Terhorst, and S. J. Morrison, "SLAM family receptors distinguish hematopoietic stem and progenitor cells and reveal endothelial niches for stem cells," Cell, vol. 121, no. 7, pp. 1109-1121, 2005. 
[64] T. Sugiyama, H. Kohara, M. Noda, and T. Nagasawa, "Maintenance of the hematopoietic stem cell pool by CXCL12CXCR4 chemokine signaling in bone marrow stromal cell niches," Immunity, vol. 25, no. 6, pp. 977-988, 2006.

[65] Y. Omatsu, T. Sugiyama, H. Kohara et al., "The essential functions of adipo-osteogenic progenitors as the hematopoietic stem and progenitor cell niche," Immunity, vol. 33, no. 3, pp. 387-399, 2010.

[66] A. I. Caplan, "Mesenchymal stem cells," Journal of Orthopaedic Research, vol. 9, no. 5, pp. 641-650, 1991.

[67] M. Owen and A. J. Friedenstein, "Stromal stem cells: marrowderived osteogenic precursors," Ciba Foundation Symposium, vol. 136, pp. 42-60, 1988.

[68] P. Bianco, P. G. Robey, and P. J. Simmons, "Mesenchymal stem cells: revisiting history, concepts, and assays," Cell Stem Cell, vol. 2, no. 4, pp. 313-319, 2008.

[69] B. Sacchetti, A. Funari, S. Michienzi et al., "Self-renewing osteoprogenitors in bone marrow sinusoids can organize a hematopoietic microenvironment," Cell, vol. 131, no. 2, pp. 324-336, 2007.

[70] M. Dominici, K. Le Blanc, I. Mueller et al., "Minimal criteria for defining multipotent mesenchymal stromal cells. The International Society for Cellular Therapy position statement," Cytotherapy, vol. 8, no. 4, pp. 315-317, 2006.

[71] W. Wagner, R. Saffrich, and A. D. Ho, "The stromal activity of mesenchymal stromal cells," Transfusion Medicine and Hemotherapy, vol. 35, no. 3, pp. 185-193, 2008.

[72] A. M. Vannucchi, L. Bianchi, C. Cellai et al., "Development of myelofibrosis in mice genetically impaired for GATA-1 expression (GATA-1low mice)," Blood, vol. 100, no. 4, pp. 1123-1132, 2002.

[73] R. A. Shivdasani and S. H. Orkin, "Erythropoiesis and globin gene expression in mice lacking the transcription factor NFE2," Proceedings of the National Academy of Sciences of the United States of America, vol. 92, no. 19, pp. 8690-8694, 1995.

[74] S. T. Avecilla, K. Hattori, B. Heissig et al., "Chemokinemediated interaction of hematopoietic progenitors with the bone marrow vascular niche is required for thrombopoiesis," Nature Medicine, vol. 10, no. 1, pp. 64-71, 2004.

[75] M. Tavassoli and M. Aoki, "Localization of megakaryocytes in the bone marrow," Blood Cells, vol. 15, no. 1, pp. 3-14, 1989.

[76] M. A. Kacena, R. A. Shivdasani, K. Wilson et al., "Megakaryocyte-osteoblast interaction revealed in mice deficient in transcription factors GATA-1 and NF-E2," Journal of Bone and Mineral Research, vol. 19, no. 4, pp. 652-660, 2004.

[77] W. A. Ciovacco, C. G. Goldberg, A. F. Taylor et al., "The role of gap junctions in megakaryocyte-mediated osteoblast proliferation and differentiation," Bone, vol. 44, no. 1, pp. 80-86, 2009.

[78] J. M. Lemieux, M. C. Horowitz, and M. A. Kacena, "Involvement of integrins $\alpha 3 \beta 1$ and $\alpha 5 \beta 1$ and glycoprotein IIb in megakaryocyte-induced osteoblast proliferation," Journal of Cellular Biochemistry, vol. 109, no. 5, pp. 927-932, 2010.

[79] R. A. Shivdasani, M. F. Rosenblatt, D. Zucker-Franklin et al., "Transcription factor NF-E2 is required for platelet formation independent of the actions of thrombopoietin/ MGDF in megakaryocyte development," Cell, vol. 81, no. 5, pp. 695704, 1995.

[80] M. A. Kacena, C. M. Gundberg, T. Nelson, and M. C. Horowitz, "Loss of the transcription factor p45 NF-E2 results in a developmental arrest of megakaryocyte differentiation and the onset of a high bone mass phenotype," Bone, vol. 36, no. 2, pp. 215-223, 2005.
[81] N. Banu and N. Williams, "Immunochemical characterisation of megakaryocyte potentiator activity from mouse bone marrow," Journal of Cellular Physiology, vol. 163, no. 3, pp. 486-492, 1995.

[82] W. S. Alexander, A. W. Roberts, N. A. Nicola, R. Li, and D. Metcalf, "Deficiencies in progenitor cells of multiple hematopoietic lineages and defective megakaryocytopoiesis in mice lacking the thrombopoietin receptor c-Mpl," Blood, vol. 87, no. 6, pp. 2162-2170, 1996.

[83] R. V. B. Emmons, D. M. Reid, R. L. Cohen et al., "Human thrombopoietin levels are high when thrombocytopenia is due to megakaryocyte deficiency and low when due to increased platelet destruction," Blood, vol. 87, no. 10, pp. 40684071, 1996

[84] C. S. Zent, J. Ratajczak, M. Z. Ratajczak, J. Anastasi, P. C. Hoffman, and A. M. Gewirtz, "Relationship between megakaryocyte mass and serum thrombopoietin levels as revealed by a case of cyclic amegakaryocytic thrombocytopenic purpura," British Journal of Haematology, vol. 105, no. 2, pp. 452458, 1999.

[85] C. A. De Graaf, M. Kauppi, T. Baldwin et al., "Regulation of hematopoietic stem cells by their mature progeny," Proceedings of the National Academy of Sciences of the United States of America, vol. 107, no. 50, pp. 21689-21694, 2010.

[86] E. Pelosi, M. Valtieri, S. Coppola et al., "Identification of the hemangioblast in postnatal life," Blood, vol. 100, no. 9, pp. 3203-3208, 2002.

[87] C. R. Cogle, D. A. Wainman, M. L. Jorgensen, S. M. Guthrie, R. N. Mames, and E. W. Scott, "Adult human hematopoietic cells provide functional hemangioblast activity," Blood, vol. 103, no. 1, pp. 133-135, 2004.

[88] E. Gunsilius, "Evidence from a leukemia model for maintenance of vascular endothelium by bone-marrow-derived endothelial cells," Advances in Experimental Medicine and Biology, vol. 522, pp. 17-24, 2003.

[89] S. B. Miller, "Prostaglandins in health and disease: an overview," Seminars in Arthritis and Rheumatism, vol. 36, no. 1, pp. 37-49, 2006.

[90] Y. Sugimoto and S. Narumiya, "Prostaglandin E receptors," Journal of Biological Chemistry, vol. 282, no. 16, pp. 1161311617, 2007.

[91] J. Hoggatt, P. Singh, J. Sampath, and L. M. Pelus, "Prostaglandin E2 enhances hematopoietic stem cell homing, survival, and proliferation," Blood, vol. 113, no. 22, pp. 54445455, 2009.

[92] C. Miyaura, M. Inada, C. Matsumoto et al., "An essential role of cytosolic phospholipase A2 $\alpha$ in prostaglandin E2-mediated bone resorption associated with inflammation," Journal of Experimental Medicine, vol. 197, no. 10, pp. 1303-1310, 2003.

[93] C. N. Pagel, S. J. Song, L. H. Loh et al., "Thrombin-stimulated growth factor and cytokine expression in osteoblasts is mediated by protease-activated receptor-1 and prostanoids," Bone, vol. 44, no. 5, pp. 813-821, 2009.

[94] C. Schmidt, G. Steinbach, R. Decking, L. E. Claes, and A. A. Ignatius, "IL-6 and PGE2 release by human osteoblasts on implant materials," Biomaterials, vol. 24, no. 23, pp. 41914196, 2003.

[95] L. M. Pelus, H. E. Broxmeyer, and M. A. S. Moore, "Regulation of human myelopoiesis by prostaglandin $\mathrm{E}$ and lactoferrin," Cell and Tissue Kinetics, vol. 14, no. 5, pp. 515-526, 1981.

[96] M. S. Kennedy, J. D. Stobo, and M. E. Goldyne, "In vitro synthesis of prostaglandins and related lipids by populations of human peripheral blood mononuclear cells," Prostaglandins, vol. 20, no. 1, pp. 135-145, 1980. 
[97] N. Hashimoto, T. Watanabe, Y. Shiratori et al., "Prostanoid secretion by rat hepatic sinusoidal endothelial cells and its regulation by exogenous adenosine triphosphate," Hepatology, vol. 21, no. 6, pp. 1713-1718, 1995.

[98] H. Rieder, G. Ramadori, K. H. Allmann, and K. H. Meyer Zum Buschenfelde, "Prostanoid release of cultured liver sinusoidal endothelial cells in response to endotoxin and tumor necrosis factor. Comparison with umbilical vein endothelial cells," Journal of Hepatology, vol. 11, no. 3, pp. 359-366, 1990.

[99] T. E. North, W. Goessling, C. R. Walkley et al., "Prostaglandin E2 regulates vertebrate haematopoietic stem cell homeostasis," Nature, vol. 447, no. 7147, pp. 1007-1011, 2007.

[100] W. Goessling, T. E. North, S. Loewer et al., "Genetic interaction of PGE2 and Wnt signaling regulates developmental specification of stem cells and regeneration," Cell, vol. 136, no. 6, pp. 1136-1147, 2009.

[101] J. Hoggatt and L. M. Pelus, "Eicosanoid regulation of hematopoiesis and hematopoietic stem and progenitor trafficking," Leukemia, vol. 24, no. 12, pp. 1993-2002, 2010.

[102] A. Kojima, M. Shiraki, and R. Takahashi, "Prostaglandin D2 is the major prostaglandin of arachidonic acid metabolism in rat bone marrow homogenate," Prostaglandins, vol. 20, no. 1, pp. 171-176, 1980.

[103] J. A. Lindgren, L. Stenke, M. Mansour et al., "Formation and effects of leukotrienes and lipoxins in human bone marrow," Journal of Lipid Mediators, vol. 6, no. 1-3, pp. 313-320, 1993.

[104] L. Stenke, M. Mansour, P. Reizenstein, and J. A. Lindgren, "Stimulation of human myelopoiesis by leukotrienes B4 and C4: interactions with granulocyte-macrophage colonystimulating factor," Blood, vol. 81, no. 2, pp. 352-356, 1993.

[105] M. G. Cipolleschi, P. D. Sbarba, and M. Olivotto, "The role of hypoxia in the maintenance of hematopoietic stem cells," Blood, vol. 82, no. 7, pp. 2031-2037, 1993.

[106] Z. Ivanovic, P. D. Sbarba, F. Trimoreau, J. L. Faucher, and V. Praloran, "Primitive human HPCs are better maintained and expanded in vitro at 1 perent oxygen than at 20 percent," Transfusion, vol. 40, no. 12, pp. 1482-1488, 2000.

[107] D. C. Chow, L. A. Wenning, W. M. Miller, and E. T. Papoutsakis, "Modeling pO2 distributions in the bone marrow hematopoietic compartment. II. Modified Kroghian models," Biophysical Journal, vol. 81, no. 2, pp. 685-696, 2001.

[108] K. Parmar, P. Mauch, J. A. Vergilio, R. Sackstein, and J. D. Down, "Distribution of hematopoietic stem cells in the bone marrow according to regional hypoxia," Proceedings of the National Academy of Sciences of the United States of America, vol. 104, no. 13, pp. 5431-5436, 2007.

[109] I. G. Winkler, V. Barbier, R. Wadley, A. C. W. Zannettino, S. Williams, and J. P. Lévesque, "Positioning of bone marrow hematopoietic and stromal cells relative to blood flow in vivo: serially reconstituting hematopoietic stem cells reside in distinct nonperfused niches," Blood, vol. 116, no. 3, pp. 375385,2010 .

[110] A. Foudi, K. Hochedlinger, D. Van Buren et al., "Analysis of histone $2 \mathrm{~B}-\mathrm{GFP}$ retention reveals slowly cycling hematopoietic stem cells," Nature Biotechnology, vol. 27, no. 1, pp. 8490, 2009.

[111] S. E. Schriner, N. J. Linford, G. M. Martin et al., "Medecine: extension of murine life span by overexpression of catalase targeted to mitochondria," Science, vol. 308, no. 5730, pp. 1909-1911, 2005.

[112] K. Ito, A. Hirao, F. Arai et al., "Regulation of oxidative stress by ATM is required for self-renewal of haematopoietic stem cells," Nature, vol. 431, no. 7011, pp. 997-1002, 2004.

[113] Y. Y. Jang and S. J. Sharkis, "A low level of reactive oxygen species selects for primitive hematopoietic stem cells that may reside in the low-oxygenic niche," Blood, vol. 110, no. 8, pp. 3056-3063, 2007

[114] K. Hosokawa, F. Arai, H. Yoshihara et al., "Function of oxidative stress in the regulation of hematopoietic stem cell-niche interaction," Biochemical and Biophysical Research Communications, vol. 363, no. 3, pp. 578-583, 2007.

[115] K. Ito, A. Hirao, F. Arai et al., "Reactive oxygen species act through p38 MAPK to limit the lifespan of hematopoietic stem cells," Nature Medicine, vol. 12, no. 4, pp. 446-451, 2006.

[116] T. Takaku, D. Malide, J. Chen, R. T. Calado, S. Kajigaya, and N. S. Young, "Hematopoiesis in 3 dimensions: human and murine bone marrow architecture visualized by confocal microscopy," Blood, vol. 116, no. 15, pp. e41-e55, 2010.

[117] C. Lo Celso, C. P. Lin, and D. T. Scadden, "In vivo imaging of transplanted hematopoietic stem and progenitor cells in mouse calvarium bone marrow," Nature Protocols, vol. 6, no. 1, pp. 1-14, 2011. 

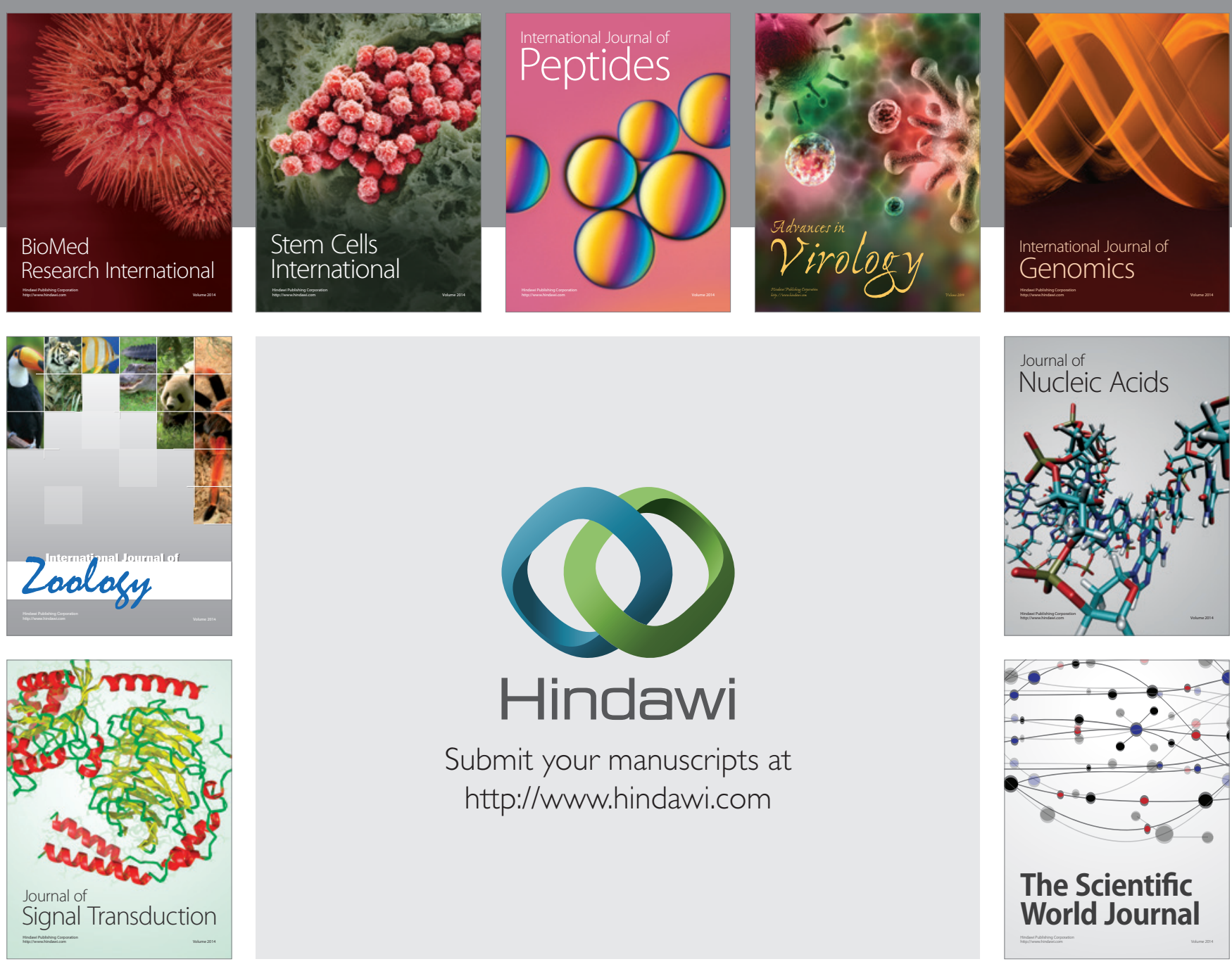

Submit your manuscripts at

http://www.hindawi.com
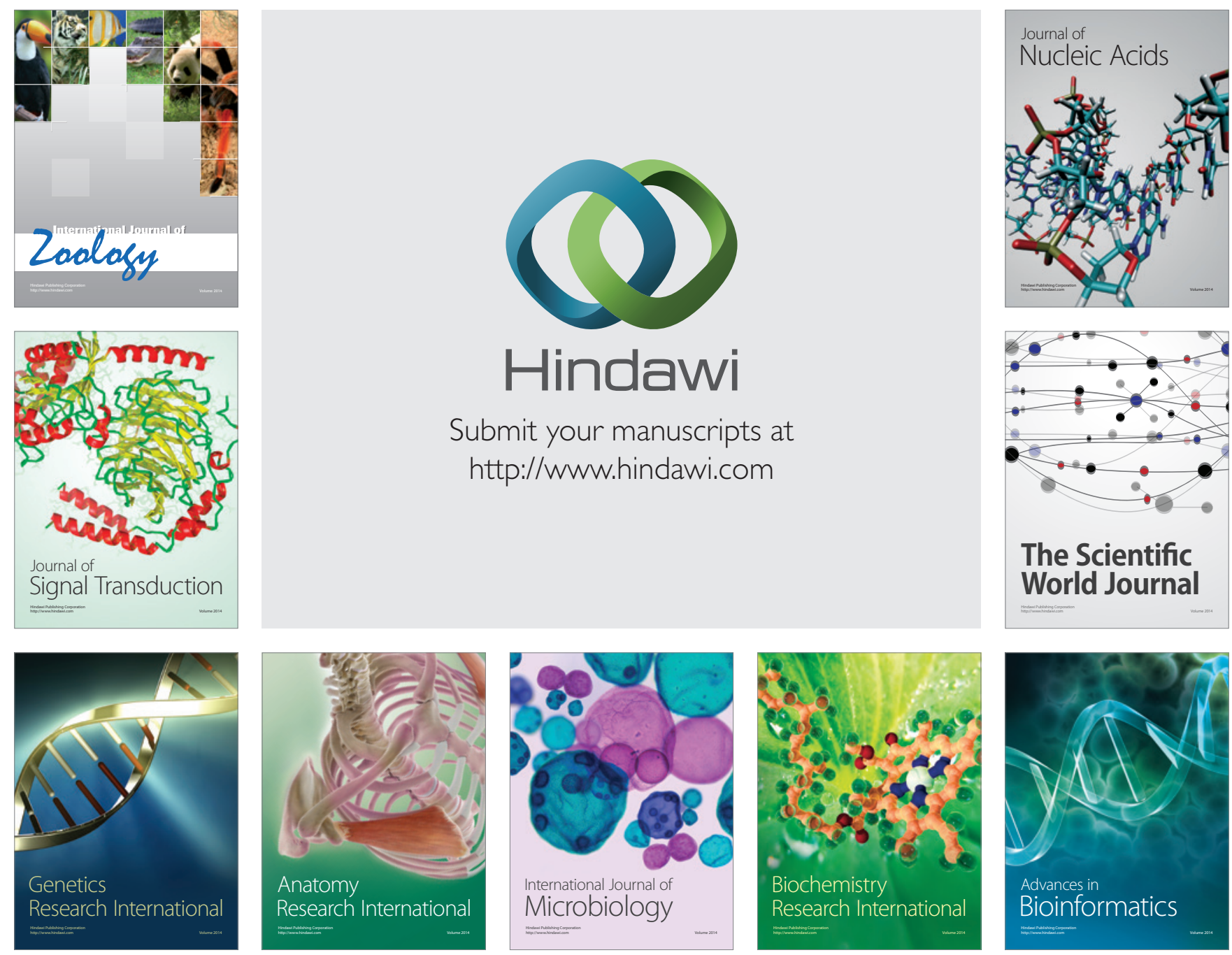

The Scientific World Journal
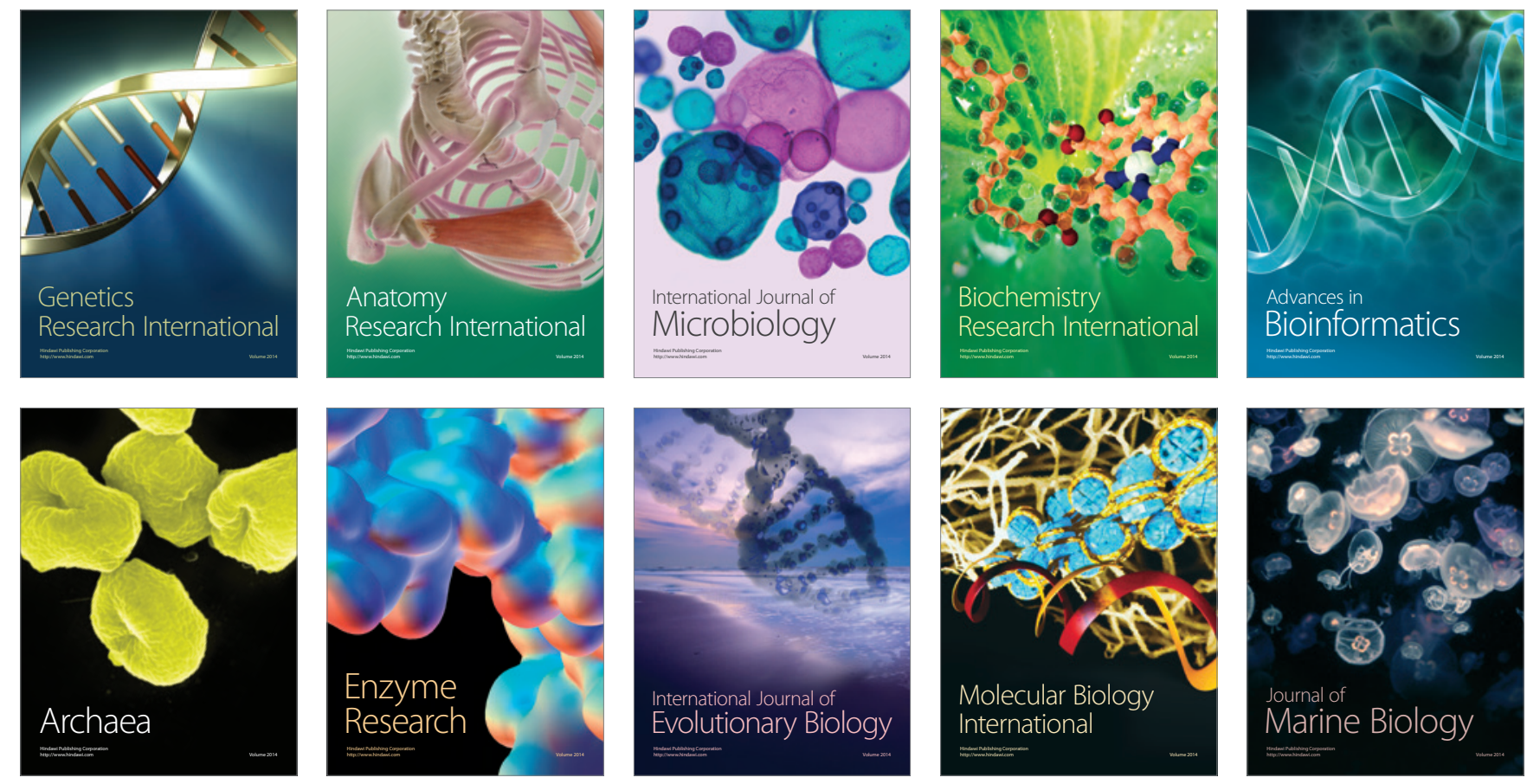